\title{
Fractional Diffusion and Medium Heterogeneity: The Case of the Continuous Time Random Walk
}

\author{
Vittoria Sposini, Silvia Vitali, Paolo Paradisi, and Gianni Pagnini
}

\begin{abstract}
In this contribution we show that fractional diffusion emerges from a simple Markovian Gaussian random walk when the medium displays a power-law heterogeneity. Within the framework of the continuous time random walk, the heterogeneity of the medium is represented by the selection, at any jump, of a different time-scale for an exponential survival probability. The resulting process is a nonMarkovian non-Gaussian random walk. In particular, for a power-law distribution of the time-scales, the resulting random walk corresponds to a time-fractional diffusion process. We relates the power-law of the medium heterogeneity to the fractional order of the diffusion. This relation provides an interpretation and an estimation of the fractional order of derivation in terms of environment heterogeneity. The results are supported by simulations.
\end{abstract}

Keywords Continuous time random walk $\cdot$ Medium heterogeneity $\cdot$ Anomalous diffusion - Time-fractional diffusion

V. Sposini

UPotsdam, Germany, and BCAM, Bilbao, Spain

Present Address:

Faculty of Physics, University of Vienna, Vienna, Austria

e-mail: vittoria.sposini@univie.ac.at

S. Vitali

BCAM, Bilbao, Spain

e-mail: svitali@bcamath.org

P. Paradisi

ISTI-CNR, Pisa, Italy

e-mail: paolo.paradisi@isti.cnr.it

G. Pagnini (殴)

BCAM and Ikerbasque, Bilbao, Spain

e-mail: gpagnini@bcamath.org

(C) The Author(s), under exclusive license to Springer Nature Switzerland AG 2021

L. Beghin et al. (eds.), Nonlocal and Fractional Operators, SEMA SIMAI Springer

Series 26, https://doi.org/10.1007/978-3-030-69236-0_14 


\section{Introduction and Motivation}

Fractional diffusion is characterized by non-Gaussian statistics and nonlinear scaling in time of the mean-squared displacement [20-22, 28]. Many different approaches have been implemented and extensively analyzed to reproduce this type of diffusion, see, e.g., [2, 9, 10]. In particular, we recall the continuous time random walk (CTRW) [11], where a power-law tailed distribution of the waiting times can be introduced to generate fractional diffusion processes. This model was proposed to describe dispersive transport of chargers in amorphous semiconductors [25]. In general, the CTRW approach can be used to model diffusion in disordered media, which are characterized by a complex trapping mechanism. Indeed, the main ingredient of CTRW models is a power-law tailed distribution for the waiting times. A direct connection between the CTRW and fractional diffusion is provided by a waiting time probability stated accordingly to the Mittag-Leffler function (ML) [7], which displays power-law tails.

Systems coming from very different fields have been studied within this framework, from geophysics to biology [17], included neurosciences where the idea of a distribution of sojourn times has been developed to describe anomalous ions diffusion in spiny dendrites $[16,26]$.

From a physical point of view, each waiting time can be related to a different probability of escaping from a trap. In this contribution, we show explicitly how complex escaping probabilities that generate fractional diffusion emerge from the combination of space heterogeneity and Markovian exponential escaping probability. The main mathematical hint behind this work is the interpretation of the ML as a weighted superposition of exponential functions [19]. In particular, we can map different trap depths into different time-scales of the exponential probability. In this way, if classical diffusion is characterised by a homogeneous landscape of traps, i.e., a constant value for the time-scale, anomalous diffusion emerges when a strong heterogeneity appears in the trap landscape, that is when a population of time-scales is introduced.

This interpretation is advantageous and more suitable for real applications, because it does not introduce complex trapping mechanism, instead it considers a large heterogeneity of simple and standard mechanisms of trapping [1]. Moreover, with the approach presented in this work, medium properties can be inferred by the statistics of the diffusing particles.

Hence, this contribution aims to provide a new and more physical interpretation of the heterogeneity described by CTRW with power-law distributed waiting times. The work is structured as follows. First, we show how the memory kernel characteristic of the CTRW model can be related to the space heterogeneity. Then we present the emergence of fractional diffusion and finally we check our results against numerical simulations. 


\section{Markovian Random Walk in a Heterogeneous Medium}

The CTRW is a successful approach to study stochastic processes $[3,5,7,8,11$, $15,18,24]$. The corresponding random walk goes on according to the following iteration procedure

$$
x_{n}=x_{n-1}+\delta x_{n}, \quad t_{n}=t_{0}+\sum_{j=1}^{n} \tau_{j}
$$

where $x_{n}$ and $x_{n-1}$ are the walker positions at the instants $t_{n}$ and $t_{n-1}$, respectively, such that the $n$-generated random jump $\delta x_{n}$ is driven by the $p d f \lambda(\delta x)$, and the corresponding random waiting-time $\tau_{n}=t_{n}-t_{n-1}$ is generated by the $p d f \psi(\tau)$. Since the probability that at least one jump is made in the temporal interval $(0, \tau)$ is given by the integral $\int_{0}^{\tau} \psi(\xi) d \xi$, then the probability that the duration of a given waiting-interval between two successive steps is strictly greater than $\tau$, i.e., the survival probability, is $\Psi(\tau)=1-\int_{0}^{\tau} \psi(\xi) d \xi$ and it holds $[15,23,27]$

$$
\psi(\tau)=-\frac{d \Psi}{d \tau}
$$

The simplest case of CTRW is the uncoupled one, i.e., the case when the jumps and the waiting times are statistically independent, and the governing equation of the process is [15]

$$
\int_{0}^{t} \Phi(t-\tau) \frac{\partial p}{\partial \tau} d \tau=-p(x, t)+\sum_{x^{\prime}} \lambda\left(x-x^{\prime}\right) p\left(x^{\prime}, t\right),
$$

with

$$
\widetilde{\Phi}(s)=\frac{1-\widetilde{\psi}(s)}{s \widetilde{\psi}(s)}=\frac{\widetilde{\Psi}(s)}{\widetilde{\psi}(s)}=\frac{\widetilde{\Psi}(s)}{1-s \widetilde{\Psi}(s)},
$$

where the symbol $\sim$ marks the Laplace transformed function and $s$ is the corresponding variable. As it follows from (3), the auxiliary function $\Phi(\tau)$ is a memory kernel. Hence, a Markovian model is obtained when $\Phi(\tau)=\delta(\tau)$, which implies that $\widetilde{\Phi}(s)=1$ and then from (4) it results $\widetilde{\Psi}(s)=\widetilde{\psi}(s)$ and also $\Psi(\tau)=\psi(\tau)$. Functions $\Psi(\tau)$ and $\psi(\tau)$ are related by formula (2), then a CTRW model is Markovian if $\Psi(\tau)=\mathrm{e}^{-\tau}$. On the contrary, when $\Psi(\tau)$ is different from an exponential function the resulting CTRW model is non-Markovian.

For the following porpuses, let us write the survival probability and waiting-time $p d f$ in the Markovian case as

$$
\Psi_{\mathrm{M}}\left(\tau^{\mathrm{M}}\right)=\mathrm{e}^{-\tau^{\mathrm{M}} / T^{0}}, \quad \psi_{\mathrm{M}}\left(\tau^{\mathrm{M}}\right)=\frac{1}{T^{0}} \mathrm{e}^{-\tau^{\mathrm{M}} / T^{0}},
$$


where the index $\mathrm{M}$ reminds the Markovian setting and the time-scale $T^{0}$ is constant if the medium is homogeneous.

Consider now a complex heterogeneous medium, such that in any position $x_{n}$ the walkers stay for a waiting time $\tau_{n}$ characterized by the medium heterogeneity. Hence, at any iteration $n$ the waiting-time $\tau_{n}$ is characterized by a local time-scale $T_{n}$. Since $T_{n}$ is the time-scale locally experienced by the particle in position $x_{n}$, the distribution of $T_{n}$ describes the spatial heterogeneity of the medium. However, if the walker lands twice in the same point then the two values of $T_{n}$ are different because independently generated. This means that the partitioning of the heterogeneity of the medium is not constant.

In this case the random walk still goes on according to the iteration procedure (1) with the same meaning for the symbols, but the probability of the waiting-time $\tau_{n}$ at the iteration $n$ is affected by the local time-scale $T_{n}$. If the motion of the walker is again assumed to be Markovian, the conditioned survival probability is

$$
\Psi\left(\tau_{n} \mid T_{n}\right)=\mathrm{e}^{-\tau_{n} / T_{n}}
$$

Comparing (5) and (6) we observe that $\tau^{\mathrm{M}} / T^{0}$ and $\tau_{n} / T_{n}$ have the same probability. By setting

$$
\frac{\tau^{\mathrm{M}}}{T^{0}}=\frac{\tau_{n}}{T_{n}}=\chi,
$$

then in formulae,

$$
\mathcal{P}\left(\frac{\tau^{\mathrm{M}}}{T^{0}}\right)=\mathcal{P}\left(\frac{\tau_{n}}{T_{n}}\right)=\mathcal{P}(\chi)=\mathrm{e}^{-\chi},
$$

and the waiting-time $\tau_{n}$ at the iteration $n$ is given by the product

$$
\tau_{n}=\frac{\tau^{\mathrm{M}}}{T^{0}} T_{n}
$$

By remembering the formulae for computing the $p d f$ of the quotient and product of independent variables, i.e.,

$$
\begin{aligned}
& \int_{0}^{\infty} b p_{A}(z b) p_{B}(b) d b, \quad Z=A / B, \\
& \int_{0}^{\infty} p_{A}\left(\frac{z}{b}\right) p_{B}(b) \frac{d b}{b}, \quad Z=A B,
\end{aligned}
$$

and by reminding that $T^{0}$ is constant and then distributed as $f\left(T_{0}\right)=\delta\left(T^{0}-T_{*}\right)$, the marginal $p d f$ of $\tau$ is

$$
\psi(\tau)=\int_{0}^{\infty} \mathrm{e}^{-\tau / T} f(T) \frac{d T}{T},
$$


and the corresponding survival probability is

$$
\Psi(\tau)=\int_{0}^{\infty} \mathrm{e}^{-\tau / T} f(T) d T
$$

The memory kernel $\Phi(t)$ in the governing equation (3) is determined by the heterogeneity and, from formula (4), its Laplace transform is

$$
\widetilde{\Phi}(s)=\frac{\int_{0}^{\infty} \frac{f(T)}{1+s T} d T}{\int_{0}^{\infty} \frac{f(T)}{1+s T} T d T}
$$

For a proper choice of the memory kernel $\Phi(t)$, the governing equation (3) results in a time-fractional diffusion equation.

\section{The Emerging of Fractional Diffusion}

In 1995 Hilfer and Anton [7] showed that CTRW is driven by the following fractional non-Markovian master equation

$$
\frac{\partial^{\beta} p}{\partial t^{\beta}}=-p(x, t)+\sum_{x^{\prime}} \lambda\left(x-x^{\prime}\right) p\left(x^{\prime}, t\right), \quad 0<\beta<1,
$$

where $\frac{\partial^{\beta}}{\partial t^{\beta}}$ can be the fractional derivative both in the Riemann-Liouville and in the Caputo sense [4], if the survival probability $\Psi(\tau)$ is a Mittag-Leffler function $[6,12$, Appendix E], i.e.,

$$
\Psi(\tau)=E_{\beta}\left(-\tau^{\beta}\right), \quad E_{\beta}(z)=\sum_{n=0}^{\infty} \frac{z^{n}}{\Gamma(\beta n+1)}, \quad z \in C, \quad 0<\beta<1 .
$$

It is well-known that a survival probability of the Mittag-Leffler type (16), when $0<\beta<1$, decreases asymptotically for $\tau \rightarrow \infty$ with the power-law $\tau^{-\beta}$ [13]. The Markovian case is recovered from the special case $E_{1}(-z)=\mathrm{e}^{-z}$. With reference to formula (13), the survival probability of Mittag-Leffler type (16) is obtained when it holds

$$
\int_{0}^{\infty} \mathrm{e}^{-t y} K_{\beta}(y) d y=E_{\beta}\left(-t^{\beta}\right), \quad 0<\beta<1,
$$

with $[4,12]$ 


$$
K_{\beta}(y)=\frac{1}{\pi} \frac{y^{\beta-1} \sin (\beta \pi)}{1+2 y^{\beta} \cos (\beta \pi)+y^{2 \beta}} .
$$

Hence, by comparing (13) and (17), the distribution of time-scales $T_{n}$ is

$$
f(T)=\frac{1}{T^{2}} K_{\beta}\left(\frac{1}{T}\right) .
$$

The asymptotic behaviour of $f(T)$ can be estimated by formula (18). Actually, it results that when $T \rightarrow \infty$ then $f(T) \sim T^{-(1+\beta)}$, and we have the following:

If the medium heterogeneity follows a distribution displaying a power-law behaviour $T^{-(1+\beta)}$, for $T \rightarrow \infty$ and $0<\beta<1$, then the random walk results in a time-fractional diffusion process of order $\beta$. This relation provides an interpretation and an estimation of the fractional order of derivation.

In the limit $\beta \rightarrow 1$, it holds $K_{\beta}(y)=\sin \pi /\left[\pi(y-1)^{2}\right] \rightarrow \delta(y-1)$ and a single time-scale follows and the Markovian case is recovered. When the distribution of the time-scales is non-stationary, i.e., $f(T)=f(T, t)$, such distribution is non-unique [19].

To conclude, when the fractional derivative in Eq. (15) is in the Caputo sense, the initial condition is $p(x, 0)=\delta(x)$, and it holds $\widehat{\lambda}(\kappa) \sim 1-\kappa^{2}$, where the symbol $\widehat{r}$ marks the Fourier transformed function and $\kappa$ the corresponding variable, then the $p d f$ of the particle displacement is

$$
p(x, t)=\frac{1}{2 t^{\beta / 2}} M_{\beta / 2}\left(\frac{|x|}{t^{\beta / 2}}\right), \quad 0<\beta<1,
$$

where $M_{v}(y), \quad 0<v<1$, is the M-Wright/Mainardi function defined as [12, Appendix F]

$$
M_{\nu}(y)=\sum_{n=1}^{\infty} \frac{(-y)^{n}}{n ! \Gamma[-v n+(1-v)]} .
$$

For completeness, the asymptotic behaviour of the $p d f$ of the particle displacement (20) is here reported. In particular, the M-Wright/Mainardi function displays stretched exponential tails in space [14]:

$$
M_{\nu}(y) \sim A_{0} Y^{\nu-1 / 2} \mathrm{e}^{-Y}, \quad y \rightarrow \infty
$$

with $A_{0}=\left[\sqrt{2 \pi}(1-v)^{v} v^{2 v-1}\right]^{-1}$ and $Y=(1-v)\left(v^{v} y\right)^{1 /(1-v)}$, and power-law decay in time:

$$
\frac{1}{r^{\nu}} M_{\nu}\left(\frac{c}{r^{v}}\right) \sim \frac{1}{r^{v}}, \quad r \rightarrow \infty
$$


This last scaling law follows from the relation between the M-Wright/Mainardi function and the extremal Lévy density [14], i.e.,

$$
\frac{1}{r^{\nu}} M_{v}\left(\frac{c}{r^{v}}\right)=\frac{r}{v c^{(1+v) / v}} L_{v}^{-v}\left(\frac{r}{c^{1 / v}}\right)
$$

\section{Numerical Simulations and Discussion}

We provide numerical results as additional proof of the result obtained in the previous section. The numerical simulations are performed in $\mathrm{C}++$ according to the scheme in (1).

For numerical purposes the waiting times $\tau_{n}$ are not extracted directly from the $p d f$ defined in (12), instead we make use of the identity in (9), where it is shown that each $\tau_{n}$ can be defined as a product of two random variables. Namely, we have

$$
\tau_{n}=\xi \cdot T_{n},
$$

where $\xi$ is an exponentially distributed random variable and $T_{n}$ is the random timescale drawn from (19). For the random generation of $\xi$ and $T_{n}$, we used the cumulative function method. That is, starting from $T_{n}$, we define $F(T)$ as the cumulative function of (19) and we obtain

$$
F(T)=\int_{0}^{T} f(t) d t=\frac{1}{\beta \pi} \arctan \left[\frac{T^{\beta}-1}{T^{\beta}+1} \tan \left(\frac{\beta \pi}{2}\right)\right]+\frac{1}{2} .
$$

Then, we consider $F(T)$ to be a function of uniform random variable between 0 and 1, i.e., $F(T)=u$, where $u \stackrel{d}{=} U(0,1)$. Finally, after substituting $u$ in (26), we get

$$
T=\left[\frac{1+A(u)}{1-A(u)}\right]^{1 / \beta}, \quad A(u)=\frac{1}{\tan (\beta \pi / 2)} \tan \left(\beta \pi\left(u-\frac{1}{2}\right)\right) .
$$

For exponentially distributed random variables, with the same procedure we can obtain the well known result

$$
\xi=-\log (u), \quad u \stackrel{d}{=} U(0,1) .
$$

Concerning the jump length, we consider a walker that, after each waiting time $\tau_{n}$, performs a jump of fixed length $j_{0}$ either to the left or to the right with equal probability, namely the Binomial random walk. In terms of CTRW notations this corresponds to a jump length $p d f$

$$
\lambda(\delta x)=\frac{1}{2}\left[\delta\left(\delta x-j_{0}\right)+\delta\left(\delta x+j_{0}\right)\right],
$$



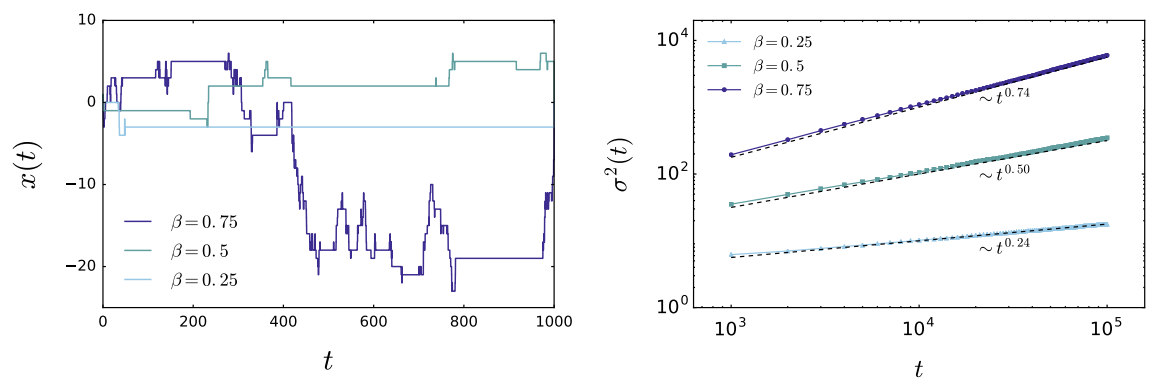

Fig. 1 Left: example of trajectories for each value of $\beta$. Right: variance for three values of $\beta=$ $0.25,0.5,0.75$. The black dashed lines represent the expected behaviour, the exponents obtained from the best fit analysis are also reported for comparison

where $\delta(x)$ is the Dirac delta function, and it is straightforward to check that

$$
\hat{\lambda}(\kappa)=\frac{1}{2}\left(\mathrm{e}^{i \kappa j_{0}}+\mathrm{e}^{-i \kappa j_{0}}\right) \sim 1-\frac{j_{0}^{2}}{2} \kappa^{2}, \quad \kappa j_{0} \ll 1 .
$$

We simulated $10^{4}$ trajectories with initial condition $x_{0}=0$, jump length $j_{0}=1$ and three different values of $\beta=0.25,0.5,0.75$. Few trajectories were stored at $10^{4}$ observation times, distributed linearly in the interval $\left[0,10^{3}\right]$. In order to perform histograms and study the $p d f \mathrm{~s}$, the positions of all trajectories were stored at 10 moments within the time interval $\left[10^{4}, 10^{5}\right]$. The variance was computed for $10^{2}$ points, distributed linearly in the time interval $\left[0,10^{5}\right]$.

In Fig. 1 we report single trajectories and the variance for three values of $\beta$. Starting from the former, we can directly observe that when $\beta$ gets closer to 1 , the waiting times become smaller and smaller. For the study of the variance we performed linear fits using the logarithm of the data. The results reported in the figure show that the subdiffussive trend of the variance is properly recovered:

$$
\left\langle x^{2}\right\rangle=\sigma^{2}(t)=\frac{j_{0}^{2}}{\Gamma(1+\beta)} t^{\beta} .
$$

In Fig. 2 the particle displacement $p d f \mathrm{~s}$ are shown. For the comparison with the analytical results we refer to the asymptotic behaviour of the $p d f$ in (20), that results in a stretched exponential, i.e.,

$$
\begin{array}{r}
p(x, t) \sim \frac{1}{\sqrt{4 \pi t^{\beta}(2-\beta)}}\left(\frac{2}{\beta}\right)^{(1-\beta) /(2-\beta)}\left(\frac{|x|}{\sqrt{t^{\beta}}}\right)^{-(1-\beta) /(2-\beta)} \\
\quad \times \exp \left[-\frac{2-\beta}{2}\left(\frac{\beta}{2}\right)^{\beta /(2-\beta)}\left(\frac{|x|}{\sqrt{t^{\beta}}}\right)^{-1 /(1-\beta / 2)}\right],
\end{array}
$$



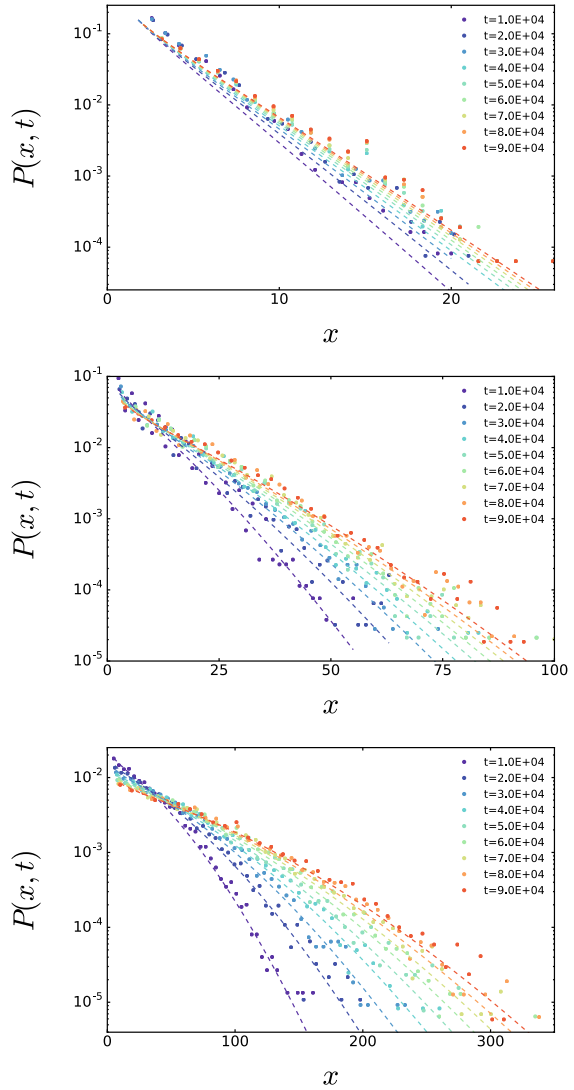

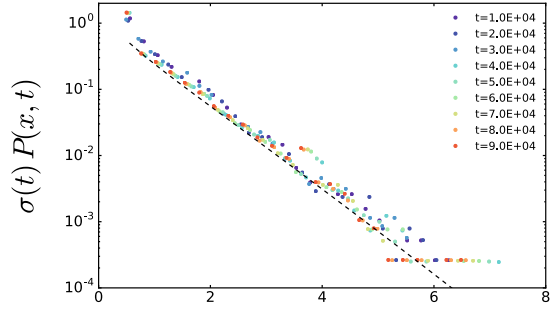

$x / \sigma(t)$
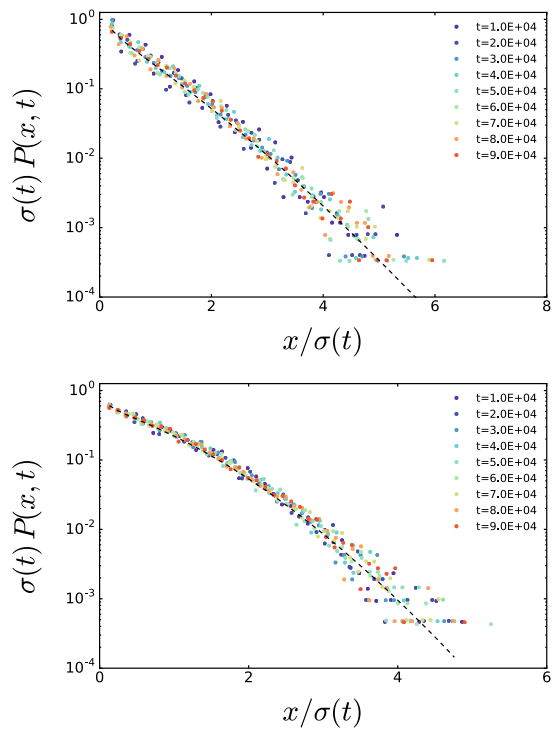

Fig. 2 Numerical results for $\beta=0.25$ (upper panel), $\beta=0.5$ (central panel) and $\beta=0.75$ (lower panel). The dashed lines on the left panels indicate the analytical result in (32); each color refers to the time in the legend, respectively. On the right panels the same quantities, rescaled by the corresponding variance at each time, are shown. The analytical behaviour in (32) rescaled by the variance is reported in black

for $|x| \gg \sqrt{t^{\beta}}$.

We observe a good agreement between numerical and analytical results. Differences are due to the fact that the jumps are performed by using the Binomial random walk, such that the convergence occurs in the diffusive limit.

Thus, we can claim that our analysis provides a simple and explicit interpretation of the CTRW with power-law distributed waiting times as a model for diffusion in heterogeneous media. Moreover, we introduced a general and operative way to directly include the heterogeneity in the diffusive model, clarifying the emergence of non-Markovian behaviour. 


\section{Conclusions}

In this contribution we show through a simple Markovian Gaussian random walk how anomalous diffusion emerges from medium heterogeneity. In particular, within the CTRW framework, the heterogeneity is represented by a random time-scale that affects the waiting-time interval at any jump. Since in any position a different timescale is considered, the distribution of the time-scales is intended as a characterisation of the spatial heterogeneity of the medium.

Actually, this time-scale is always independently generated, which means that in the same position, but at different instants, different time-scales may be experienced by the walkers. That is to say that the heterogeneity of the medium is not constant.

For a proper distribution of the time-scales with a power-law behaviour for large values, the evolution equation of the density of the walkers' displacement emerges to be a time-fractional diffusion equation.

The present derivation of a time-fractional diffusion process, in terms of medium heterogeneity through a population of time-scales (19), complements the results derived in Ref. [19]. The study is supported by numerical simulations of the process. In this respect it is reported that, unlike other algorithms for fractional processes from the CTRW, the waiting-time distribution is not chosen a priori but generated through the proposed mechanism based on random time-scales applied to a Markovian Gaussian random walk. This mechanism defines a framework which is able to reproduce a large class of diffusion processes, that comes from any possible medium heterogeneity without changing the algorithm behind the single trap mechanisms, which remains a Markovian process. The fractional process emerges as a particular case in which strong heterogeneity, characterized by power law tail in the time scales distribution, is considered.

To conclude, we report that through Eq. (17) we define a relation between the medium heterogeneity and the fractional order of the diffusion equation, by the introduction of a population of timescales. Within this approach, the power law tail of the distribution of the timescales can be estimated from the fractional order of the diffusion process. This relation establishes an interpretation of the fractional order that goes beyond the one given in the standard CTRW, providing a physical interpretation of the variability of the waiting times in terms of environment heterogeneity without modifying the physical mechanism behind the single trapping event.

Acknowledgements This research was supported by the Basque Government through the BERC 2018-2021 program, and by the Spanish Ministry of Economy and Competitiveness MINECO through BCAM Severo Ochoa excellence accreditation SEV-2017-0718. 


\section{References}

1. Camboni, F., Sokolov, I.M.: Normal and anomalous diffusion in random potential landscapes. Phys. Rev. E 85, 050104(R) (2012)

2. Di Tullio, F., Paradisi, P., Spigler, R., Pagnini, G.: Gaussian processes in complex media: new vistas on anomalous diffusion. Front. Phys. 7, 123 (2019)

3. Fulger, D., Scalas, E., Germano, G.: Monte Carlo simulation of uncoupled continuous-time random walks yielding a stochastic solution of the space-time fractional diffusion equation. Phys. Rev. E 77, 021122 (2008)

4. Gorenflo, R., Mainardi, F.: Fractional calculus: integral and differential equations of fractional order. In: Carpinteri, A., Mainardi, F. (eds.) Fractals and Fractional Calculus in Continuum Mechanics, pp. 223-276. Springer, Wien and New York (1997)

5. Gorenflo, R., Mainardi, F.: Some recent advances in theory and simulation of fractional diffusion processes. J. Comput. Appl. Math. 229(2), 400-415 (2009)

6. Haubold, H.J., Mathai, A.M., Saxena, R.K.: Mittag-Leffler functions and their applications. J. Appl. Math. 2011, 298,628 (2011)

7. Hilfer, R., Anton, L.: Fractional master equations and fractal time random walks. Phys. Rev. E 51(2), R848-R851 (1995)

8. Klafter, J., Blumen, A., Shlesinger, M.F.: Stochastic pathway to anomalous diffusion. Phys. Rev. A 35(7), 3081-3085 (1987)

9. Klafter, J., Lim, S.C., Metzler, R.: Fractional Dynamics: Recent Advances. World Scientific, Singapore (2011)

10. Klages, R., Radons, G., Sokolov, I.M. (eds.): Anomalous Transport: Foundations and Applications. Wiley-VCH Verlag GmbH \& Co. KGaA, Weinheim (2008)

11. Kutner, R., Masoliver, J.: The continuous time random walk, still trendy: fifty-year history, state of art and outlook. Eur. Phys. J. B 90, 50 (2017)

12. Mainardi, F.: Fractional Calculus and Waves in Linear Viscoelasticity. Imperial College Press (2010)

13. Mainardi, F., Gorenflo, R.: On Mittag-Leffler-type functions in fractional evolution processes. J. Comput. Appl. Math. 118(1-2), 283-299 (2000)

14. Mainardi, F., Luchko, Y., Pagnini, G.: The fundamental solution of the space-time fractional diffusion equation. Fract. Calc. Appl. Anal. 4(2), 153-192 (2001)

15. Mainardi, F., Raberto, M., Gorenflo, R., Scalas, E.: Fractional calculus and continuous-time finance II: the waiting-time distribution. Phys. A 287(3-4), 468-481 (2000)

16. Méndez, V., Iomin, A.: Comb-like models for transport along spiny dendrites. Chaos Solitons Fract. 53(Supplement C), 46-51 (2013)

17. Metzler, R., Klafter, J.: The restaurant at the end of the random walk: recent developments in fractional dynamics descriptions of anomalous dynamical processes. J. Phys. A: Math. Theor. 37(31), R161-R208 (2004)

18. Montroll, E.W., Weiss, G.H.: Random walks on lattices. II. J. Math. Phys. 6(2), 167-181 (1965)

19. Pagnini, G.: Short note on the emergence of fractional kinetics. Phys. A 409, 29-34 (2014)

20. Reynolds, A.: Liberating Lévy walk research from the shackles of optimal foraging. Phys. Life Rev. 14, 59-83 (2015)

21. Sahimi, M.: Heterogeneous Materials I. Linear Transport and Optical Properties, 1 edn. Interdisciplinary Applied Mathematics. Springer, Berlin (2003)

22. Santamaria, F., Wils, S., Schutter, E.D., Augustine, G.J.: Anomalous diffusion in Purkinje cell dendrites caused by spines. Neuron 52(4), 635-648 (2006)

23. Scalas, E., Gorenflo, R., Mainardi, F.: Fractional calculus and continuous-time finance. Phys. A 284, 376-384 (2000)

24. Scalas, E., Gorenflo, R., Mainardi, F.: Uncoupled continuous-time random walks: solution and limiting behavior of the master equation. Phys. Rev. E 69, 011107 (2004)

25. Scher, H., Montroll, E.W.: Anomalous transit-time dispersion in amorphous solids. Phys. Rev. B 12, 2455-2477 (1975) 
26. Vitali, S., Mainardi, F., Castellani, G.: Emergence of fractional kinetics in spiny dendrites. Fractal Fract. 2(1), 6 (2018)

27. Weiss, G.H.: Aspects and Applications of the Random Walk. North-Holland, Amsterdam (1994)

28. Witzel, P., Götz, M., Lanoiselée, Y., Franosch, T., Grebenkov, D.S., Heinrich, D.: Heterogeneities shape passive intracellular transport. Biophys. J. 117(2), 203-213 (2019) 